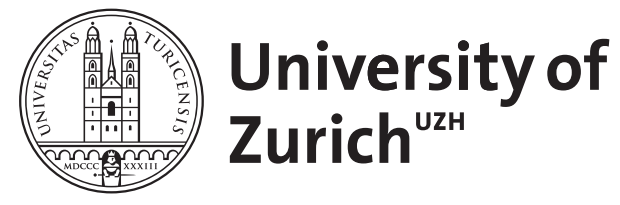

\title{
Products of hyperbolic metric spaces
}

Foertsch, T; Schroeder, Viktor

\begin{abstract}
Let (X i d i ), i=1,2, be proper geodesic hyperbolic metric spaces. We give a general construction for a 'hyperbolic product' $\mathrm{X} 1 \times \mathrm{h} \mathrm{X} 2$ which is itself a proper geodesic hyperbolic metric space and examine its boundary at infinity.
\end{abstract}

DOI: https://doi.org/10.1023/B:GEOM.0000006539.14783.aa

Posted at the Zurich Open Repository and Archive, University of Zurich ZORA URL: https://doi.org/10.5167/uzh-21874

Journal Article

Accepted Version

Originally published at:

Foertsch, T; Schroeder, Viktor (2003). Products of hyperbolic metric spaces. Geometriae Dedicata, 102(1):197-212.

DOI: https://doi.org/10.1023/B:GEOM.0000006539.14783.aa 


\title{
Products of hyperbolic metric spaces
}

\author{
Thomas Foertsch*, $\quad$ Viktor Schroeder
}

\begin{abstract}
Let $\left(X_{i}, d_{i}\right), i=1,2$, be proper geodesic hyperbolic metric spaces. We give a general construction for a "hyperbolic product" $X_{1} \times_{h} X_{2}$ which is itself a proper geodesic hyperbolic metric space and examine its boundary at infinity.
\end{abstract}

\section{Introduction}

Let $\left(X_{i}, d_{i}\right), i=1,2$, be proper geodesic hyperbolic metric spaces (for definitions see Section 2). We give a general construction for a "hyperbolic product" $X_{1} \times_{h} X_{2}$ which is itself a proper geodesic hyperbolic metric space.

This construction only depends on chosen basepoints $z_{i} \in X_{i}$ or on points $u_{i} \in \partial X_{i}$, where $\partial X_{i}$ is the boundary at infinity.

For given points $z_{i} \in X_{i}$ consider the set

$$
Y:=\left\{\left(x_{1}, x_{2}\right) \in X_{1} \times X_{2} \mid d_{1}\left(x_{1}, z_{1}\right)=d_{2}\left(x_{2}, z_{2}\right)\right\} \subset X_{1} \times X_{2}
$$

with the induced product metric

$$
d_{2}\left(\left(x_{1}, x_{2}\right),\left(y_{1}, y_{2}\right)\right)=\left(d_{1}^{2}\left(x_{1}, y_{1}\right)+d_{2}^{2}\left(x_{2}, y_{2}\right)\right)^{\frac{1}{2}} .
$$

Let $d: Y \times Y \longrightarrow \mathbb{R}^{+}$be the corresponding interior metric

$$
d(x, y):=\inf \{L(c) \mid c \text { connecting } x \text { to } y \text { in } Y\}
$$

where $L(c)$ denotes the length of $c$ in $\left(Y, d_{e}\right)$.

We call $(Y, d)$ the hyperbolic product of $\left(X_{1}, z_{1}\right)$ and $\left(X_{2}, z_{2}\right)$ and denote it also by

$$
Y=\left(X_{1}, z_{1}\right) \times_{h}\left(X_{2}, z_{2}\right) .
$$

This notion is justified by the following

*supported by SNF Grant 21 - 58938.99

2000 Mathematics Subject Classification. Primary 53C21 
Theorem 1 Let $X_{i}, i=1,2$, be proper geodesic hyperbolic spaces and $z_{i} \in X_{i}$, $i=1,2$. Then $Y=\left(X_{1}, z_{1}\right) \times_{h}\left(X_{2}, z_{2}\right)$ is also a proper geodesic hyperbolic space and $\partial Y$ is naturally homeomorphic to $\partial X_{1} \times \partial X_{2}$.

The construction can be carried over in the limit case that the points $z_{i}$ tend to infinity. This limit case seems to be of particular interest.

Let $u_{i} \in \partial X_{i}$ be given. These points give rise to Busemann functions $B_{i}$ : $X_{i} \longrightarrow \mathbb{R}$. Define now

$$
Y:=\left\{\left(x_{1}, x_{2}\right) \in X_{1} \times X_{2} \mid B_{1}\left(x_{1}\right)=B_{2}\left(x_{2}\right)\right\} \subset X_{1} \times X_{2}
$$

and consider as above the interior metric $d$ on $Y$.

We call $(Y, d)$ the hyperbolic product of $\left(X_{1}, B_{1}\right)$ and $\left(X_{2}, B_{2}\right)$, denote it by

$$
Y=\left(X_{1}, B_{1}\right) \times_{h}\left(X_{2}, B_{2}\right),
$$

and obtain the

Theorem 2 Let $X_{i}, i=1,2$, be proper geodesic hyperbolic spaces and $B_{i}$ : $X_{i} \longrightarrow \mathbb{R}$ Busemann functions on $X_{i}$. Then $Y=\left(X_{1}, B_{1}\right) \times_{h}\left(X_{2}, B_{2}\right)$ is also a proper geodesic hyperbolic space and $\partial Y$ is naturally homeomorphic to the smashed product $\partial X_{1} \wedge \partial X_{2}$.

Remark 1 i) The smashed product $\wedge$ is a standard construction for pointed topological spaces. Let $\left(U_{1}, u_{1}\right),\left(U_{2}, u_{2}\right)$ be two pointed spaces then the smashed product $U_{1} \wedge U_{2}$ is defined as $U_{1} \times U_{2} / U_{1} \vee U_{2}$, where $U_{1} \times U_{2}$ is the usual product and

$$
U_{1} \vee U_{2}=\left(\left\{u_{1}\right\} \times U_{2}\right) \cup\left(U_{2} \times\left\{u_{2}\right\}\right) \subset U_{1} \times U_{2}
$$

is the wedge product canonically embedded in $U_{1} \times U_{2}$. Thus $U_{1} \wedge U_{2}$ is obtained from $U_{1} \times U_{2}$ by collapsing $U_{1} \vee U_{2}$ to a point. For example $S^{m} \wedge S^{n}=S^{m+n}$.

ii) In [BrFa] the authors proved that the hyperbolic product of real hyperbolic spaces $\left(\mathbb{H}^{m_{1}}, u_{1}\right) \times_{h}\left(\mathbb{H}^{m_{2}}, u_{2}\right)$, with $u_{i}$ in the ideal boundary, is isometric to a real hyperbolic space $\mathbb{H}^{m_{1}+m_{2}-1}$.

In [FS] the authors proved that, more generally, the hyperbolic product $(Y, d)$ of Hadamard manifolds of pinched negative sectional curvature $-b^{2} \leq$ $K_{i} \leq-a^{2}<0$ is hyperbolic. This was done by showing that there exists a metric on $Y$ that is bilipschitz to the one induced by the canonical embedding $i: Y \longrightarrow X$ and that carries pinched negative sectional curvature. For related results also see [L1], [L2], [F1] and [F2].

iii) Instead of the Euclidean product metric $d_{e}$ on $Y$ we could also take e.g. the maximum metric

$$
d_{m}\left(\left(x_{1}, x_{2}\right),\left(y_{1}, y_{2}\right)\right):=\max \left\{d_{1}\left(x_{1}, y_{1}\right), d_{2}\left(x_{2}, y_{2}\right)\right\}
$$


and the corresponding inner metric $d^{\prime}$ on $Y$. Note that $d_{e}$ and $d_{m}$ and therefore also $d$ and $d^{\prime}$ are bilipschitz related.

The following holds in general: If $(Y, d)$ is a proper geodesic metric space and $d^{\prime}$ is an other interior metric on $Y$ which is bilipschitz related to $d$, then $\left(Y, d^{\prime}\right)$ also is a proper metric space which implies that it is geodesic since $(Y, d)$ is a length space (see e.g. Theorem 2.5.23 in [BuBuI]). In addition $(Y, d)$ is hyperbolic if and only if $\left(Y, d^{\prime}\right)$ is hyperbolic and in that case $\partial(Y, d)$ is homeomorphic to $\partial\left(Y, d^{\prime}\right)$.

For technical reasons we use in our proof the metric $d_{m}$ on $X_{1} \times X_{2}$.

iv) Finally note that the hyperbolic product can similar be defined for finitely many factors and the analogue of Theorems 1 and 2 hold in that case.

Outline of the paper:

In Section 2 we collect the necessary results on hyperbolic metric spaces. In Sections 3 and 4 we give a proof of Theorem 2. At the end of Section 3 we indicate the necessary changes for the situation of Theorem 1.

Acknowledgment: We want to thank Urs Lang for useful discussions.

\section{Preliminaries}

\subsection{Hyperbolicity}

A metric space $(X, d)$ is called geodesic, if any two points $x, y \in X$ can be joined by a geodesic segment $\overline{x y}$ that is the image of a geodesic path $\gamma_{x y}$ : $[0, d(x, y)] \longrightarrow X$ from $x$ to $y$ which is parameterized by arclength.

A geodesic metric space is called $\delta$-hyperbolic if for any triangle with geodesic sides in $X$ each side is contained in the $\delta$ - neighborhood of the union of the two other sides.

The space is called hyperbolic if it is $\delta$-hyperbolic for some $\delta \geq 0$.

Let $X$ be a metric space and $x, y, z \in X$. Then there exist unique $a, b, c \in \mathbb{R}_{0}^{+}$ such that

$$
d(x, y)=a+b, \quad d(x, z)=a+c \quad \text { and } \quad d(y, z)=b+c .
$$

In fact those numbers are given through

$$
a=(y \cdot z)_{x}, \quad b=(x \cdot z)_{y}, \quad \text { and } \quad c=(x \cdot y)_{z},
$$

where for instance

$$
(y \cdot z)_{x}=\frac{1}{2}[d(y, x)+d(z, x)-d(y, z)] .
$$

In the case that $X$ is geodesic we may consider a geodesic triangle $\overline{x y} \cup \overline{x z} \cup \overline{y z} \subset$ $X$, where for example $\overline{x y}$ denotes a geodesic segment connecting $x$ to $y$. Given 
such a triangle we denote by $\tilde{x}=\gamma_{x y}(a)$ the unique point on $\overline{y z}$ satisfying $d(\tilde{x}, y)=(x \cdot z)_{y}$ and in the same way we define $\tilde{y} \in \overline{x z}$ and $\tilde{z} \in \overline{x y}$.

Note that for $X$ being a tree all these points coincide, i.e. $\tilde{x}=\tilde{y}=\tilde{z}$. In general an upper bound for the distances of these points measures the hyperbolicity of $(X, d)$.

Lemma 1 i) If $(X, d)$ is $\delta$-hyperbolic, then

$$
d(z, \tilde{z}) \leq c+2 \delta, \quad d\left(\gamma_{x y}(t), \gamma_{x z}(t)\right) \leq 4 \delta \quad \forall t \in[0, a]
$$

and the points $\tilde{x}, \tilde{y}, \tilde{z}$ have pairwise distance $\leq 4 \delta$.

ii) A metric space $(X, d)$ is hyperbolic if and only if there exists a $\delta^{\prime} \in \mathbb{R}_{0}^{+}$such that given any geodesic triangle $\overline{x y} \cup \overline{x z} \cup \overline{y z} \subset X$ the points $\tilde{x} \in \overline{y z}, \tilde{y} \in \overline{x z}$ and $\tilde{z} \in \overline{x y}$ as defined above have distance less than $\delta^{\prime}$ to each other.

Proof: i) By $\delta$-hyperbolicity $d(\tilde{z}, \overline{x z}) \geq \delta$ or $d(\tilde{z}, \overline{y z}) \geq \delta$. By triangle inequality we have in the first case $d(\tilde{z}, \tilde{y}) \leq 2 \delta$ and hence $d(\tilde{z}, z) \leq c+2 \delta$. The other case is similar.

Assume that there is $t_{0} \in[0, a]$ with $d\left(\gamma_{x y}\left(t_{0}\right), \gamma_{x z}\left(t_{0}\right)\right)>4 \delta$, then $d\left(\gamma_{x y}\left(t_{0}-\delta\right), \gamma_{x z}\left(t_{0}-\delta\right)\right)>2 \delta$ which implies $d\left(\gamma_{x y}\left(t_{0}-\delta\right), \overline{x z}\right)>\delta$ and by hyperbolicity $d\left(\gamma_{x y}\left(t_{0}-\delta\right), \overline{y z}\right)<\delta$. Let $p \in \overline{y z}$ be a point of minimal distance to $\gamma\left(t_{0}-\delta\right)$. By triangle inequality $d(p, y) \geq b$ and hence $d(p, z) \leq c$. Thus

$$
\begin{aligned}
a+c=d(x, z) & \leq\left(t_{0}-\delta\right)+d\left(\gamma_{x y}\left(t_{0}-\delta\right), p\right)+d(p, z) \\
& <a+c ;
\end{aligned}
$$

a contradiction. Since the corresponding estimate holds for the other sides as well the points $\tilde{x}, \tilde{y}$ and $\tilde{z}$ have pairwise distance $\leq 4 \delta$.

For $i$ ) compare to Proposition III.1.17 in [BriH].

\section{$2.2 T$-functions}

Definition 1 Let $\alpha, \omega \in \mathbb{R}$ and $I:=[\alpha, \omega]$.

i) A function $f: I \longrightarrow \mathbb{R}$ is called a T-function if $f$ is continuous and there exists $\alpha+a \in[\alpha, \omega]$ such that the restrictions $\left.f\right|_{(\alpha, \alpha+a)}$ and $\left.f\right|_{(\alpha+a, \omega)}$ are differentiable with

$$
\left.f^{\prime}\right|_{(\alpha, \alpha+a)} \equiv-1 \quad \text { and }\left.\quad f^{\prime}\right|_{(\alpha+a, \omega)} \equiv 1 .
$$

ii) A function $f: I \longrightarrow \mathbb{R}$ is called a $\delta$-T-function, $\delta \in \mathbb{R}_{0}^{+}$, if there exists a $T$-function $g: I \longrightarrow \mathbb{R}$ such that $\|f-g\|_{\text {sup }}<\delta$.

iii) Let $X$ be a geodesic metric space. A function $f: X \longrightarrow \mathbb{R}$ is called a $\delta$ - $T$ function, if for any geodesic segment $\gamma:[\alpha, \omega] \longrightarrow \mathbb{R}$ the function $f \circ \gamma$ is a $\delta$-T-function. 
Remark 2 It is straight forward to check that

i) Every T-function $f$ is convex and Lipschitz with Lipschitz constant $l=1$.

ii) For $[\alpha, \omega] \subset \mathbb{R}$ and $t_{1}, t_{2} \in \mathbb{R}$ with $\left|t_{1}-t_{2}\right| \leq|\alpha-\omega|$ there exists a unique $T$-function with $f(\alpha)=t_{1}$ and $f(\omega)=t_{2}$. Indeed there are unique $a, b, c \in$ $\mathbb{R}_{0}^{+}$such that $a+b=|\alpha-\omega|, a+c=t_{1}, b+c=t_{2}$. These are given via

$$
\begin{aligned}
a & =\frac{1}{2}\left(|\alpha-\omega|+t_{1}-t_{2}\right), \\
b & =\frac{1}{2}\left(|\alpha-\omega|+t_{2}-t_{1}\right), \\
c & =\frac{1}{2}\left(t_{1}+t_{2}-|\alpha-\omega|\right) .
\end{aligned}
$$

Now $f$ satisfies

$$
f(\alpha)=t_{1}, \quad f(\alpha+a)=c, \quad f(\omega)=t_{2} .
$$

iii) $f$ is a ( $\delta$-)T-function $\Longrightarrow f+$ const is a $(\delta$ - $) T$-function.

iv) A limit of a sequence of ( $\delta$-)T-functions is a $(\delta$-)T-function.

Lemma 2 Let $X$ be a geodesic metric space. Then the following are equivalent:

i) $X$ is hyperbolic.

ii) There exists a $\delta \in \mathbb{R}^{+}$such that for all $x \in X$ the function

$$
d_{x}: X \longrightarrow \mathbb{R}, \quad y \longmapsto d(x, y)
$$

is a $\delta$-T-function.

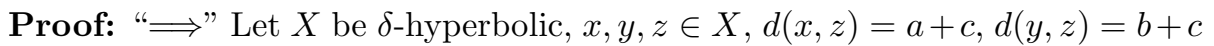
and $\gamma:[0, a+b] \longrightarrow \overline{x y}$ an arc length parameterized geodesic connecting $x$ to $y$.

Now consider the $T$-function $f:[0, a+b] \longrightarrow \mathbb{R}^{+}$determined by

$$
f(0)=d(z, \gamma(0))=a+c \quad \text { and } \quad f(a+b)=d(z, \gamma(a+b))=b+c .
$$

Note that $f(a)=c$ and $\left(d_{z} \circ \gamma\right)(a) \leq c+4 \delta$ by Lemma $\left.1 i i\right)$.

From the fact that $d_{z} \circ \gamma:[0, a+b] \longrightarrow \mathbb{R}^{+}$is 1-Lipschitz it immediately follows that

$$
\left\|d_{z} \circ \gamma-f\right\|_{\text {sup }} \leq 4 \delta .
$$

Hence $d_{z} \circ \gamma$ is a $4 \delta$ - $T$-function.

"£" Let now $X$ satisfy condition $i i$ ). We show that $X$ is hyperbolic using the criterion of Lemma $1 i$ ): 
For $x, y, z \in X$ and geodesic segments $\overline{x y}, \overline{x z}$ and $\overline{y z}$ connecting these points, condition $i i)$ yields $c \leq d(z, \tilde{z}) \leq c+\delta$. We now consider the geodesic triangle $\overline{x \tilde{z}} \cup \overline{z \tilde{z}} \cup \overline{x z}$, where $\overline{x \tilde{z}} \subset \overline{x y}$ and $\overline{z \tilde{z}}$ is any geodesic segment connecting $z$ to $\tilde{z}$. For $\hat{z} \in \overline{x z}$ satisfying $d(z, \hat{z})=(x \cdot \tilde{z})_{z}$ condition $\left.i i\right)$ gives $d(\tilde{z}, \hat{z})<\delta$. Furthermore one has $c<d(z, \hat{z})<c+\frac{\delta}{2}$ and therefore $d(\tilde{y}, \hat{z})<\frac{\delta}{2}$. Thus we achieve

$$
d(\tilde{z}, \tilde{y}) \leq d(\tilde{z}, \hat{z})+d(\hat{z}, \tilde{y}) \leq \delta+\frac{\delta}{2}=\frac{3}{2} \delta .
$$

The same argument of course yields $d(\tilde{z}, \tilde{x}) \leq \frac{3}{2} \delta$ and $d(\tilde{y}, \tilde{x}) \leq \frac{3}{2} \delta$. By Lemma $1 \mathrm{ii}$ ) we obtain the result.

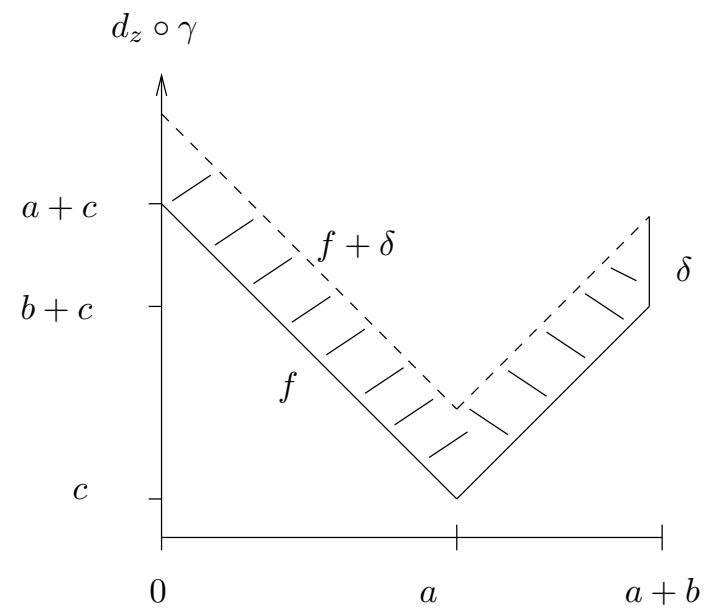

Figure 1: The graph of $d_{z} \circ \gamma$ lies in the region between the graphs of $f$ and $f+\delta$.

\subsection{The boundary at infinity and Busemann functions}

We associate to a hyperbolic space $(X, d)$ a boundary $\partial X$ at infinity. There are different descriptions in the literature (see e.g. $[\mathrm{BeKa}]$ ) all of which coincide for proper geodesic hyperbolic spaces.

We choose a basepoint $z \in X$. We say that a sequence $\left\{x^{k}\right\}_{k \in \mathbb{N}}$ of points in $X$ converges to infinity, if

$$
\liminf _{k, l \longrightarrow \infty}\left(x^{k} \cdot x^{l}\right)_{z}=\infty
$$

Two sequences $\left\{x^{k}\right\}_{k \in \mathbb{N}}$ and $\left\{y^{k}\right\}_{k \in \mathbb{N}}$ converging to infinity are equivalent, $\left\{x^{k}\right\}_{k \in \mathbb{N}} \sim\left\{y^{k}\right\}_{k \in \mathbb{N}}$ if

$$
\liminf _{k, l \longrightarrow \infty}\left(x^{k} \cdot y^{l}\right)_{z}=\infty
$$


One shows that $\sim$ is an equivalence relation and defines $\partial X$ as the set of equivalence classes. We write $\left[\left\{x^{k}\right\}\right] \in \partial X$ for the corresponding class. One can also show that for every $x \in X$ and $v \in \partial X$ there is a geodesic ray $\gamma_{x v}:[0, \infty] \longrightarrow X$ parameterized by arclength with $\gamma_{x v}(0)=x$ and $\left[\left\{\gamma_{x v}(k)\right\}\right]=v$.

For $v \in \partial X$ and $r>0$ one defines

$U(v, r):=\left\{w \in \partial X \mid \exists\left\{x^{k}\right\},\left\{y^{k}\right\}\right.$ s.t. $\left.\left[\left\{x^{k}\right\}\right]=v,\left[\left\{y^{k}\right\}\right]=w, \liminf _{k, l \rightarrow \infty}\left(x^{k} \cdot y^{l}\right)_{z} \geq r\right\}$.

On $\partial X$ we consider the topology generated by $U(v, r), v \in \partial X, r>0$.

If $X$ is proper, $\partial X$ is a compact topological space. We now also fix a basepoint $u \in \partial X$ and a geodesic ray $\gamma_{z u}$ from $z$ to $u$. The function $B: X \longrightarrow \mathbb{R}$, $B(x):=\lim _{t \rightarrow \infty}\left[d\left(x, \gamma_{z u}(t)\right)-t\right]$ is called the Busemann function associated to $\gamma_{z u} . B$ is a $\delta$-T-function as a limit of $\delta$-T-functions.

Definition 2 A geodesic ray $\gamma:[0, \infty) \longrightarrow X$ is called a $B$-ray if and only if $\gamma$ is parameterized by arc length and

$$
B(\gamma(t))=B(\gamma(0))-t \quad \forall t \in[0, \infty) .
$$

By a standard limit argument we obtain the

Lemma 3 Let $X$ be a complete, locally compact, hyperbolic metric space and $B$ a Busemann function on $X$. Then for every $x \in X$ there exists a $B$-ray $\gamma$ with $\gamma(0)=x$.

Let now $v \in \partial X \backslash\{u\}$ and consider $B$-rays $\gamma_{\gamma_{z v}(t)}$ starting at $\gamma_{z v}(t)$. Then these rays subconverge as sets to a geodesic from $v$ to $u$ and by suitable reparameterization we obtain the existence of a geodesic $\gamma_{v u}: \mathbb{R} \longrightarrow X$ with

$$
B\left(\gamma_{v u}(t)\right)=-t, \quad\left[\left\{\gamma_{v u}(k)\right\}\right]=u \quad \text { and } \quad\left[\left\{\gamma_{v u}(-k)\right\}\right]=v .
$$

The hyperbolicity of $X$ also implies that there exists a constant $C$ (depending on $u, v, z)$ such that

$$
d\left(\gamma_{z}(t), \gamma_{v u}(t)\right) \leq C \quad \text { and } \quad d\left(\gamma_{z v}(t), \gamma_{v u}(-t)\right) \leq C \quad \forall t \geq 0 .
$$

Let now $x, y \in X$ and $\gamma_{x}, \gamma_{y}$ be $B$-rays starting at $x, y$. Then $\gamma_{x}, \gamma_{y}$ and a geodesic segment $\overline{x y}$ form an ideal triangle with vertices $x, y$ and $u=\gamma_{z u}(\infty) \in$ $\partial X$. We want to look for points $\tilde{u}, \tilde{x}, \tilde{y}$ as for finite triangles. Clearly there are $a, b \geq 0$ such that $d(x, y)=a+b$ and $B\left(\gamma_{x}(a)\right)=B\left(\gamma_{x}(b)\right)$. Indeed

$$
\begin{aligned}
a=(y \cdot B)_{x} & :=\frac{1}{2}(d(y, x)+B(x)-B(y)) \text { and } \\
b & =(x \cdot B)_{y}:=\frac{1}{2}(d(x, y)+B(y)-B(x)) .
\end{aligned}
$$

Let $\tilde{u} \in \overline{x y}$ be the point with $d(x, \tilde{u})=a$ and let $\tilde{y}=\gamma_{x}(a), \tilde{x}=\gamma_{y}(b)$. 

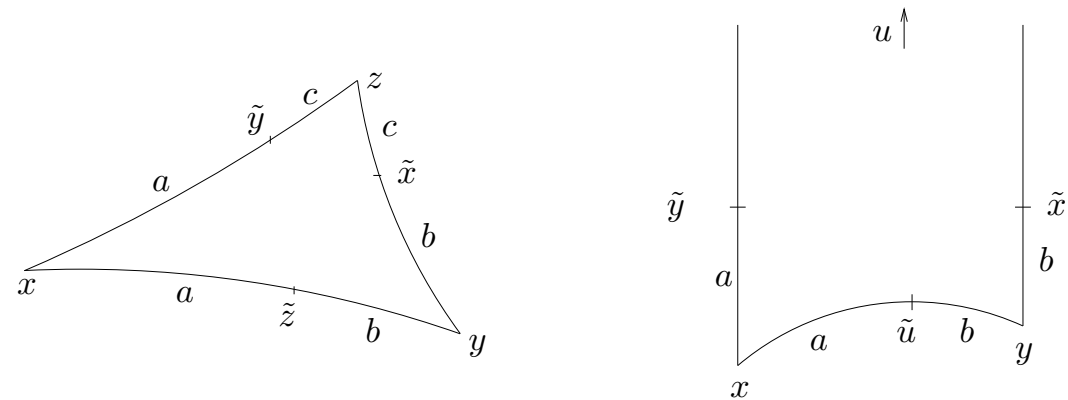

Figure 2: Triangle and ideal triangle in the hyperbolic space.

Lemma 4 i) The points $\tilde{x}, \tilde{y}$ and $\tilde{u}$ have pairwise distance $\leq 8 \delta$.

ii) For all $t \geq 0$ it holds $d\left(\gamma_{x}(a+t), \gamma_{y}(b+t)\right) \leq 8 \delta$.

Proof: Let $B(x)=\lim _{t \rightarrow \infty}\left(d\left(x, \gamma_{z u}(t)\right)-t\right), z_{i}=\gamma_{z u}(i), i \in \mathbb{N}$, and consider the triangle $x, y, z_{i}$ with corresponding values $a_{i}, b_{i}, c_{i} \in \mathbb{R}^{+}$as well as points

$$
\hat{z}_{i}=\gamma_{x y}\left(a_{i}\right), \quad \hat{y}_{i}=\gamma_{x z_{i}}\left(a_{i}\right) \quad \text { and } \quad \hat{x}_{i}=\gamma_{y z_{i}}\left(b_{i}\right) .
$$

Clearly one has $a_{i} \longrightarrow a$ and $b_{i} \longrightarrow b$.

Consider also the triangles $x, \gamma_{x}\left(a_{i}\right), z_{i}$. Note that $\left|d\left(z_{i}, \gamma_{x}\left(a_{i}\right)\right)-d\left(z_{i}, \hat{y}_{i}\right)\right| \longrightarrow 0$ which implies by Lemma $1 \mathrm{ii}$ )

$$
\limsup _{i \longrightarrow \infty} d\left(\gamma_{x}\left(a_{i}\right), \tilde{y}_{i}\right) \leq 2 \delta .
$$

In the same way we obtain

$$
\limsup _{i \longrightarrow \infty} d\left(\gamma_{y}\left(b_{i}\right), \tilde{x}_{i}\right) \leq 2 \delta .
$$

Since $d\left(\tilde{x}_{i}, \tilde{y}_{i}\right) \leq 4 \delta$ by Lemma 1 we obtain $\left.i\right)$.

The proof of $i$ i) is similar.

We need the following

Lemma 5 Let $x, y \in X$ and $\sigma:[0, d(x, y)] \longrightarrow X$ a curve parameterized by arclength such that $\sigma(0)=x$ and $d(\sigma(d(x, y)), y) \leq R$. Then

$$
d\left(\gamma_{x y}(t), \sigma(t)\right) \leq \frac{3}{2} R+4 \delta \quad \forall t \in[0, d(x, y)] .
$$

Proof: For fixed $t \in[0, d(x, y)]$ consider the triangle $x, y, z:=\sigma(t)$. Since $d(x, z) \leq t$ and $d(z, y) \leq d(x, y)-t+R$ we have $c=(x \cdot y)_{z} \leq \frac{R}{2}$ and hence there exists $\tilde{z}=\gamma_{x y}\left(t^{\prime}\right)$ with $d(z, \tilde{z}) \leq \frac{R}{2}+4 \delta$. Note that $t^{\prime}=d(x, z)-c$ and $d(x, y)-t^{\prime}=d(z, y)-c$. Thus $\left|t-t^{\prime}\right| \leq R$ from which the claim follows. 


\subsection{A Morse estimate}

We need an estimate whose proof is similar to the proof of the Morse inequality.

Lemma 6 Let $(X, d)$ be $\delta$-hyperbolic, $x, y \in X$ and $\gamma:[0,1] \longrightarrow X$ be a continuous path from $x$ to $y$. If there exists a point $p=\gamma_{x y}\left(s_{0}\right) \in \overline{x y}$ such that $d(p, \gamma(t)) \geq R$ for all $t \in[0,1]$ and $R>90 \delta$, then

$$
L(\gamma) \geq d(x, y)+\frac{1}{20 \delta} R^{2} .
$$

Proof: Define $a(t):=(y \cdot \gamma(t))_{x} \in[0, d(x, y)]$. Since $a$ is continuous, $a(0)=0$ and $a(1)=d(x, y)$ there are $0<t_{-}<t_{+}<1$ such that

$a\left(t_{-}\right)=s_{0}-\frac{R}{2}, \quad a\left(t_{+}\right)=s_{0}+\frac{R}{2}, \quad a(t) \in\left[s_{0}-\frac{R}{2}, s_{0}+\frac{R}{2}\right] \quad \forall t_{-}<t<t_{+}$.

Choose $k+1:=\left[\frac{R}{8 \delta}\right]+1$ points $s_{1} \leq \ldots \leq s_{k+1} \in\left[s_{0}-\frac{R}{2}, s_{0}+\frac{R}{2}\right]$ such that $\left|s_{i+1}-s_{i}\right| \geq 12 \delta$ and let $t_{i} \in\left[t_{-}, t_{+}\right]$be points $t_{1} \leq t_{2} \leq \ldots \leq t_{k+1}$ with $a\left(t_{i}\right)=s_{i}$. Now

$$
L(\gamma) \geq d\left(x, \gamma\left(t_{-}\right)\right)+\sum_{i=1}^{k} d\left(\gamma\left(t_{i+1}\right), \gamma\left(t_{i}\right)\right)+d\left(\gamma\left(t_{+}\right), y\right) .
$$

By construction

$$
d\left(x, \gamma\left(t_{-}\right)\right) \geq a\left(t_{-}\right) \quad \text { and } \quad d\left(y, \gamma\left(t_{+}\right)\right) \geq d(x, y)-a\left(t_{-}\right) .
$$

Thus $d\left(x, \gamma\left(t_{-}\right)\right)+d\left(\gamma\left(t_{+}\right), y\right) \geq d(x, y)-R$.

Let $q_{i} \in \overline{x y}$ be a point such that $d\left(\gamma\left(t_{i}\right), q_{i}\right)=d\left(\gamma\left(t_{i}\right), \overline{x y}\right)$. Since $d_{\gamma\left(t_{i}\right)} \circ \gamma_{x y}$ is a $4 \delta$-T-function by Lemma 2 , a minimum of this function is assumed in distance $\leq 4 \delta$ of the corresponding $T$-function. Thus $d\left(q_{i}, \gamma_{x y}\left(s_{i}\right)\right) \leq 4 \delta$, which implies $d\left(q_{i}, q_{i+1}\right) \geq 4 \delta$ since $d\left(\gamma_{x, y}\left(s_{i+1}\right), \gamma_{x y}\left(s_{i}\right)\right) \geq 12 \delta$.

By Lemma 8.4.23 in [BuBuI] we obtain

$$
d\left(\gamma\left(t_{i+1}, \gamma\left(t_{i}\right)\right) \geq d\left(\gamma\left(t_{i+1}\right), q_{i+1}\right)+d\left(\gamma\left(t_{i}\right), q_{i}\right)-4 \delta .\right.
$$

Since $d\left(\gamma\left(t_{i}\right), p\right) \geq R$ and $d\left(q_{i}, p\right) \leq \frac{R}{2}+4 \delta$ we obtain $d\left(\gamma\left(t_{i+1}\right), \gamma\left(t_{i}\right)\right) \geq$ $R-12 \delta$. Thus

$$
\begin{aligned}
L(\gamma) & \geq d(x, y)+\left[\frac{R}{12 \delta}\right](R-12 \delta)-R \\
& \geq d(x, y)+\frac{1}{20 \delta} R^{2}
\end{aligned}
$$

for $R \geq 90 \delta$. 


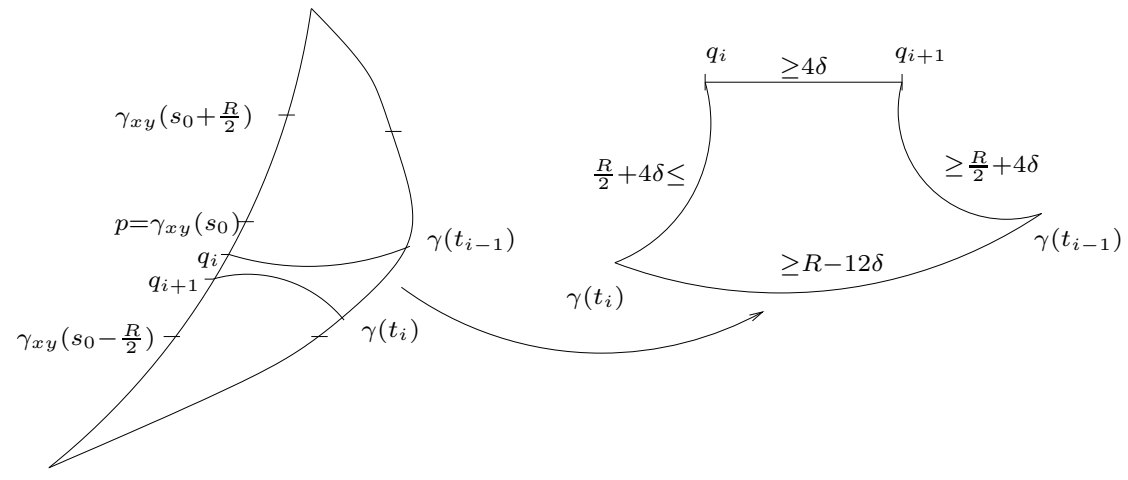

Figure 3: This figure shows how Lemma 8.4.23 in [BuBuI] is applied to the segments $\left.\gamma_{x y}\right|_{\left[s_{i}, s_{i+1}\right]}$ and $\left.\gamma\right|_{t_{i}, t_{i+1}}$ in the proof of Lemma 6 .

\section{The hyperbolic product}

In this section we prove the first part of Theorem 2, which is equal to Proposition 3 .

Let $\left(X_{i}, d_{i}\right)$ be $\delta_{i}$-hyperbolic spaces, $i=1,2$, and $\delta:=\max \left\{\delta_{1}, \delta_{2}\right\}$. Let further $B_{i}: X_{i} \longrightarrow \mathbb{R}$ be Busemann functions on $X_{i}$. We study the set

$$
Y:=\left\{\left(x_{1}, x_{2}\right) \in X_{1} \times X_{2} \mid B_{1}\left(x_{1}\right)=B_{2}\left(x_{2}\right)\right\} .
$$

On $Y$ we consider the maximum metric $d_{m}: Y \times Y \longrightarrow \mathbb{R}$,

$$
d_{m}\left(\left(x_{1}, x_{2}\right),\left(x_{1}^{\prime}, x_{2}^{\prime}\right)\right):=\max \left\{d_{1}\left(x_{1}, x_{1}^{\prime}\right), d_{2}\left(x_{2}, x_{2}^{\prime}\right)\right\} .
$$

For our use of $d_{m}$ instead of $d_{e}$ compare Remark 1 iii) in the Introduction.

Let $p, p^{\prime} \in Y$. We first construct two curves in $Y$ between $p$ and $p^{\prime}$, the $\Gamma$-curve $\Gamma_{p p^{\prime}}$ and the continuous $\Gamma$-curve $\Gamma_{p p^{\prime}}^{c}$.

The advantage of $\Gamma_{p p^{\prime}}$ is that this curve is conceptually easy to understand. However, $\Gamma_{p p^{\prime}}$ is in general not continuous, $\Gamma_{p p^{\prime}}^{c}$ is a continuous variation of $\Gamma_{p p^{\prime}}$.

Let $p=\left(p_{1}, p_{2}\right), p^{\prime}=\left(p_{1}^{\prime}, p_{2}^{\prime}\right)$ and $\gamma_{i}, \gamma_{i}^{\prime}$ be $B_{i}$-rays starting at $p_{i}, p_{i}^{\prime}$. Let further $\gamma, \gamma^{\prime}: \mathbb{R}_{0}^{+} \longrightarrow Y$ be the geodesic rays

$$
\gamma(t)=\left(\gamma_{1}(t), \gamma_{2}(t)\right), \quad \gamma^{\prime}(t)=\left(\gamma_{1}^{\prime}(t), \gamma_{2}^{\prime}(t)\right) .
$$

These geodesics are parameterized by constant speed 1 . We set

$$
\begin{aligned}
a_{i}:=\left(p_{i}^{\prime} \cdot B_{i}\right)_{p_{i}}=\frac{1}{2}\left(d\left(p_{i}, p_{i}^{\prime}\right)+B_{i}\left(p_{i}\right)-B_{i}\left(p_{i}^{\prime}\right)\right) \text { and } \\
b_{i}:=\left(p_{i} \cdot B_{i}\right)_{p_{i}^{\prime}}=\frac{1}{2}\left(d\left(p_{i}, p_{i}^{\prime}\right)+B_{i}\left(p_{i}^{\prime}\right)-B_{i}\left(p_{i}\right)\right),
\end{aligned}
$$


such that $a_{i}+b_{i}=d_{i}\left(p_{i}, p_{i}^{\prime}\right)$. Let $a:=\max \left\{a_{1}, a_{2}\right\}$ and $b:=\max \left\{b_{1}, b_{2}\right\}$, then $a+b=d_{m}\left(p, p^{\prime}\right)$. We define by a slight abuse of notation

$$
\Gamma=\Gamma_{p p^{\prime}}:=\left.\left.\gamma\right|_{[0, a]} * \gamma^{\prime}\right|_{[0, b]} ^{-1} .
$$

Note that $\Gamma_{p p^{\prime}}$ is not necessarily continuous, since $\gamma(a)=\left(\gamma_{1}(a), \gamma_{2}(a)\right)$ is not necessarily equal to $\gamma^{\prime}(b)=\left(\gamma_{1}^{\prime}(b), \gamma_{2}^{\prime}(b)\right)$. However, $d_{i}\left(\gamma_{i}(a), \gamma_{i}^{\prime}(b)\right) \leq 8 \delta_{i}$ by Lemma 4.

The curve $\Gamma_{p p^{\prime}}^{c}$ is a continuous modification of $\Gamma_{p p^{\prime}}$ and defined as

$$
\Gamma_{p p^{\prime}}^{c}:=\left.\left.\gamma\right|_{[0, a+2 \delta]} * \Gamma_{1} * \Gamma_{2} * \gamma^{\prime}\right|_{[0, b+2 \delta]} ^{-1},
$$

where $\Gamma_{1}$ is a continuous curve in $Y$ form $\left(\gamma_{1}(a+2 \delta), \gamma_{2}(a+2 \delta)\right)$ to $\left(\gamma_{1}^{\prime}(b+\right.$ $\left.2 \delta), \gamma_{2}(a+2 \delta)\right)$ and $\Gamma_{2}$ a continuous curve in $Y$ from $\left(\gamma_{1}^{\prime}(b+2 \delta), \gamma_{2}(a+2 \delta)\right)$ to $\left(\gamma_{1}^{\prime}(b+2 \delta), \gamma_{2}^{\prime}(b+2 \delta)\right)$ given in the following way:

Let $\eta_{1}:\left[\alpha_{1}, \beta_{1}\right] \longrightarrow X_{1}$ be a geodesic from $\gamma_{1}(a+2 \delta)$ to $\gamma_{1}^{\prime}(b+2 \delta)$. Note that $L\left(\eta_{1}\right) \leq 8 \delta$ and $B_{1}\left(\eta_{1}\left(\alpha_{1}\right)\right)=B_{2}\left(\eta_{1}\left(\alpha_{2}\right)\right)$. Since $B_{1}$ is 1-Lipschitz and a $4 \delta$ - $T$-function we obtain

$$
B_{1}\left(\eta_{1}(t)\right) \leq B_{1}\left(\eta_{1}\left(\alpha_{1}\right)\right)+2 \delta=B_{1}\left(p_{1}\right)-a .
$$

Thus $-B_{1}\left(\eta_{1}(t)\right)+B_{1}\left(p_{1}\right) \geq a \geq 0$ and

$$
\Gamma_{1}(t)=\left(\eta_{1}(t), \gamma_{2}\left(-B_{1}\left(\eta_{1}(t)\right)+B_{1}\left(p_{1}\right)\right)\right)
$$

is well defined. By construction $\Gamma_{1}(t) \in Y$ and $L_{d_{m}}\left(\Gamma_{1}\right) \leq 8 \delta$.

In a similar way one constructs $\Gamma_{2}$.

We can easily estimate the length of $\Gamma_{p p^{\prime}}^{c}$ and obtain the

Lemma 7 Given two points $p, p^{\prime} \in Y$ the continuous curve $\Gamma^{c}=\Gamma_{p p^{\prime}}^{c}$ has length

$$
L\left(\Gamma^{c}\right) \leq d_{m}\left(p, p^{\prime}\right)+20 \delta .
$$

This immediately implies the

Proposition 1 Given two points $p, p^{\prime} \in Y$, it holds

$$
d_{m}\left(p, p^{\prime}\right) \leq d\left(p, p^{\prime}\right) \leq d_{m}\left(p, p^{\prime}\right)+20 \delta .
$$

Thus $\left(Y, d_{m}\right)$ and $(Y, d)$ are quasi-isometric and hence bilipschitz on a large scale. But also on a local scale they induce the same topology:

Lemma 8 The metrics $d$ and $\left.d_{m}\right|_{Y}$ induce the same topology on $Y$.

Proof: We need to show that

$$
\left.\lim _{i \longrightarrow \infty} d_{m}\right|_{Y}\left(y_{i}, y_{0}\right)=0 \quad \Longrightarrow \quad \lim _{i \longrightarrow \infty} d\left(y_{i}, y_{0}\right)=0 .
$$

Thus it suffices to prove the 
Sublemma 1 Let $\left\{y_{i}\right\}_{i \in \mathbb{N}}$ be a sequence in $Y$ that converges to $y_{0} \in Y$ with respect to $\left.d_{e}\right|_{Y}$. Then for all $\epsilon>0$ there exists $\rho(\epsilon)>0$ such that for all $y_{i} \in Y$ satisfying $\left.d_{e}\right|_{Y}\left(y_{i}, y_{0}\right)<\rho$ there exists a curve $\Gamma_{i}$ in $Y$ connecting $y_{i}$ to $y_{0}$ of length $L\left(\Gamma_{i}\right) \leq \epsilon$.

A sequence $\left\{y_{i}\right\}_{i \in \mathbb{N}}=\left\{\left(y_{i 1}, y_{i 2}\right)\right\}_{i \in \mathbb{N}}$ in $Y \subset X_{1} \times X_{2}$ converges with respect to $\left.d_{m}\right|_{Y}$ if and only if the sequences $\left\{y_{i j}\right\}_{i \in \mathbb{N}}$ in $X_{j}$ converge with respect to $d_{j}$, $j=1,2$.

Define

$$
K_{j}:=\left\{\gamma\left(\frac{\epsilon}{4}\right) \mid \gamma \text { is a } B_{j} \text {-ray with } \gamma(0)=y_{0 j}\right\}
$$

and let $\gamma_{i j}$ be $B_{j}$-rays with $\gamma_{i j}(0)=y_{i j}$. The local compactness of $X_{j}$ implies the

Claim: There exists $\rho>0$ such that $d_{j}\left(y_{i j}, y_{0 j}\right)<\delta$ implies $d\left(\gamma_{i j}\left(\frac{\epsilon}{4}\right), K_{j}\right) \leq \frac{\epsilon}{8}$.

Let now $d_{m}\left(y_{i}, y_{0}\right)<\rho$, then $d_{j}\left(y_{i j}, y_{0 j}\right)<\rho$ and thus by the claim there are $B_{j}$ rays $\gamma_{j}$ starting at $y_{0 j}$ such that $d\left(\gamma_{i j}\left(\frac{\epsilon}{4}\right), \gamma_{j}\left(\frac{\epsilon}{4}\right)\right)<\frac{\epsilon}{8}$. Similar to the construction of $\Gamma_{p p^{\prime}}^{c}$ one now finds a continuous path $\tilde{\Gamma}^{c}$ in $Y$ connecting successively the points

$$
\left(y_{i 1}, y_{i 2}\right),\left(\gamma_{i 1}\left(\frac{\epsilon}{8}\right), \gamma_{i 2}\left(\frac{\epsilon}{8}\right)\right), \quad\left(\gamma_{1}\left(\frac{\epsilon}{8}\right), \gamma_{i 2}\left(\frac{\epsilon}{8}\right)\right),\left(\gamma_{1}\left(\frac{\epsilon}{8}\right), \gamma_{2}\left(\frac{\epsilon}{8}\right)\right), \quad\left(y_{01}, y_{02}\right)
$$

of length

$$
\tilde{\Gamma}^{c}<\frac{\epsilon}{4}+\frac{\epsilon}{8}+\frac{\epsilon}{8}+\frac{\epsilon}{4}=\epsilon .
$$

Corollary $1(Y, d)$ is locally compact, complete and hence proper and geodesic.

Proof: Since the Busemann functions $b_{i}, i=1,2$, are continuous, $Y$ is a closed subset of the locally compact space $\left(X_{1} \times X_{2}, d_{m}\right)$ and therefore locally compact itself when endowed with the induced metric $\left.d_{m}\right|_{Y}$. Thus from Lemma 8 it follows that $(Y, d)$ is also locally compact.

Every Cauchy-sequence in $(Y, d)$ is a Cauchy-sequence in $\left(Y,\left.d_{m}\right|_{Y}\right)$. But $\left(Y,\left.d_{m}\right|_{Y}\right)$ is complete and therefore the Cauchy-sequence converges in $\left(Y,\left.d_{m}\right|_{Y}\right)$. Now the proof of Lemma 8 yields convergence in $(Y, d)$. Hence $(Y, d)$ is complete.

Finally every locally compact, complete length space is proper and geodesic (see e.g. Proposition I.3.7. in $[\mathrm{BriH}])$.

Let $p, p^{\prime} \in Y$ and let

$$
\sigma:\left[0, d\left(p, p^{\prime}\right)\right] \longrightarrow Y, \quad \sigma(t)=\left(\sigma_{1}(t), \sigma_{2}(t)\right)
$$

be a unit speed geodesic from $p$ to $p^{\prime}$. We want to compare $\sigma$ with the curve $\Gamma\left(p, p^{\prime}\right)$. To have the same domain, we modify $\Gamma_{p p^{\prime}}$ a little: Let $a, b$ as above 
and

$$
\begin{aligned}
a^{*} & =a+\frac{1}{2}\left(d\left(p, p^{\prime}\right)-d_{m}\left(p, p^{\prime}\right)\right), \\
b^{*} & =b+\frac{1}{2}\left(d\left(p, p^{\prime}\right)-d_{m}\left(p, p^{\prime}\right)\right) .
\end{aligned}
$$

We define $\Gamma^{*}=\Gamma_{p p^{\prime}}^{*}:\left[0, d\left(p, p^{\prime}\right)\right] \longrightarrow Y$ via

$$
\Gamma_{p p^{\prime}}^{*}(t):=\left.\left.\gamma\right|_{\left[0, a^{*}\right]} * \gamma^{\prime}\right|_{\left[0, b^{*}\right]}-1
$$

and prove the

Proposition 2 For $\sigma$ and $\Gamma^{*}$ as above it holds

$$
d\left(\sigma(t), \Gamma^{*}(t)\right) \leq 500 \delta .
$$

To simplify the arguments, we will assume:

(a) $d\left(p_{1}, p_{1}^{\prime}\right) \geq d\left(p_{2}, p_{2}^{\prime}\right)$ which implies $a=a_{1}$ and $b=b_{1}$,

(b) $B_{i}\left(p_{i}\right)=0, B_{i}\left(\gamma_{i}(t)\right)=-t$, and $B_{i}\left(\gamma_{i}^{\prime}(t)\right)=-t+(b-a)$.

We can assume this without loss of generality: $(a)$ by interchanging the factors and $(b)$ by adding the same constant to both Busemann functions.

The first step in the proof of Proposition 2 is the

Lemma 9 There exists $t_{0} \in\left[0, d\left(p, p^{\prime}\right)\right]$ with $d_{1}\left(\sigma_{1}\left(t_{0}\right), \gamma_{1}(a)\right) \leq 30 \delta$.

Proof: Consider the ideal triangle $p_{1}, p_{1}^{\prime}, u_{1}=\gamma_{1}(\infty)=\gamma_{1}^{\prime}(\infty)$ in $X_{1}$ with points $\tilde{p}_{1}^{\prime}=\gamma_{1}(a), \tilde{p}_{1}=\gamma^{\prime}(b), \tilde{u}_{1}=\gamma_{p_{1}, p_{1}^{\prime}}(a) \in \overline{p_{1}, p_{1}^{\prime}}$ of pairwise distance $\leq 8 \delta$. Choose $t_{0}$ such that

$$
M:=d\left(\sigma_{1}\left(t_{0}\right), \tilde{u}_{1}\right)=\min _{t} d\left(\sigma_{1}(t), \tilde{u}_{1}(t)\right) .
$$

By Lemma 6 we have $L_{d_{1}}\left(\sigma_{1}\right) \geq(a+b)+\frac{1}{20 \delta} M^{2}$. Since $L_{d_{1}}\left(\sigma_{1}\right) \leq L_{d_{m}}(\sigma) \leq$ $(a+b)+20 \delta$ we obtain $M \leq 20 \delta$ and thus the result.

We decompose $\sigma$ into two pieces $\sigma=\bar{\alpha} * \bar{\beta}$ where $\bar{\alpha}=\left.\sigma\right|_{\left[0, t_{0}\right]}$ and $\bar{\beta}=\left.\sigma\right|_{\left[t_{0}, d\left(p, p^{\prime}\right)\right]}$, write $\bar{\alpha}=\left(\bar{\alpha}_{1}, \bar{\alpha}_{2}\right)$ and $\bar{\beta}=\left(\bar{\beta}_{1}, \bar{\beta}_{2}\right)$ and prove the

Lemma 10 With the notation above it holds

$$
\left|L\left(\bar{\alpha}_{i}\right)-a\right| \leq 50 \delta \quad \text { and } \quad\left|L\left(\bar{\beta}_{i}\right)-b\right| \leq 50 \delta \text { for } i=1,2 .
$$


Proof: With $M$ as in Lemma 9 we compute

$L\left(\bar{\alpha}_{i}\right) \geq\left|B_{i}\left(\sigma_{i}\left(t_{0}\right)\right)-B_{i}\left(p_{i}\right)\right|=\left|B_{1}\left(\sigma_{1}\left(t_{0}\right)\right)\right| \geq a-M-8 \delta \geq a-30 \delta$.

and

$$
L\left(\bar{\beta}_{i}\right) \geq\left|B_{i}\left(\sigma_{i}\left(t_{0}\right)\right)-B_{i}\left(p_{i}^{\prime}\right)\right|=\left|B_{1}\left(\sigma_{1}\left(t_{0}\right)\right)-(b-a)\right| \geq b-30 \delta .
$$

Since $L\left(\bar{\alpha}_{i}\right)+L\left(\bar{\beta}_{i}\right) \leq a+b+20 \delta$ by Lemma 7 we obtain the result.

Lemma 11 With $t_{0}$ as in Lemma 9 it holds $d_{2}\left(\sigma_{2}\left(t_{0}\right), \gamma_{2}(a)\right) \leq 100 \delta$.

Proof: Consider the ideal triangle $p_{2}, q=\sigma_{2}\left(t_{0}\right)=\bar{\alpha}_{2}\left(t_{0}\right), u_{2}=\gamma_{2}(\infty)$ in $X_{2}$ with corresponding points $\tilde{q}=\gamma_{2}\left(\left(q \cdot B_{2}\right)_{p_{2}}\right), \tilde{u}_{2}, \tilde{p}_{2}$. Since

$$
\left(q \cdot B_{2}\right)_{p_{2}}=\frac{1}{2}\left(d\left(p_{2}, \bar{\alpha}_{2}\left(t_{0}\right)\right)+B_{2}\left(p_{2}\right)-B_{2}\left(\bar{\alpha}_{2}\left(t_{0}\right)\right)\right)
$$

and $\left|L\left(\bar{\alpha}_{1}-a\right)\right| \leq 50 \delta,\left|B_{2}\left(p_{2}\right)-B_{2}\left(\bar{\alpha}_{e}\left(t_{0}\right)\right)\right| \geq a-30 \delta$ by Lemma 10 , we see

$$
\left|\left(q \cdot B_{2}\right)_{p_{2}}-a\right| \leq 40 \delta
$$

and

$$
d\left(\tilde{u}_{2}, \tilde{p}_{2}\right)=\frac{1}{2}\left(d\left(p_{2}, \bar{\alpha}_{2}\left(t_{0}\right)\right)+B_{2}\left(\bar{\alpha}_{2}\left(t_{0}\right)\right)-B_{2}\left(p_{2}\right)\right) \leq 40 \delta .
$$

Together with Lemma 4 we get the estimate.

Proof of Proposition 2: Lemmata 9, 11 and Proposition 1 imply that $d\left(\sigma\left(t_{0}\right), \gamma(a)\right) \leq$ $120 \delta$. Combining some triangle inequalities we obtain $\left|t_{0}-a\right| \leq 150 \delta$ and

$$
d\left(\sigma\left(a^{*}\right), \gamma\left(a^{*}\right)\right) \leq 300 \delta, \quad d\left(\sigma\left(a^{*}\right), \gamma\left(b^{*}\right)\right) \leq 300 \delta .
$$

Together with Lemma 5 we obtain Proposition 2.

Proposition $3(Y, d)$ is hyperbolic.

Proof: By Lemma 2 it suffices to show that there exists a $\Delta$ such that for all $q, p, p^{\prime} \in Y$ and all minimal geodesics $\sigma:\left[0, d\left(p, p^{\prime}\right)\right] \longrightarrow Y$ from $p$ to $p^{\prime}$ the function $t \longmapsto d(q, \sigma(t))$ is a $\Delta$-T-function. By Propositions 1 and 2 it suffices to show that there exists $\Delta$ such that for all $q, p, p^{\prime}$ the function

$$
\left[a^{*}, b^{*}\right] \longrightarrow \mathbb{R} \quad t \longmapsto d_{m}\left(q, \Gamma^{*}(t)\right)
$$

is a $\Delta$-T-function, where $\Gamma^{*}=\left.\left.\gamma\right|_{[0, a *]} * \gamma^{\prime}\right|_{\left[0, b^{*}\right]} ^{-1}$ as defined above. Define $f, g:\left[a^{*}, b^{*}\right] \longrightarrow \mathbb{R}$ via

$$
f(t):=d_{1}\left(q_{1}, \Gamma_{1}^{*}(t)\right) \quad \text { and } \quad g(t):=d_{2}\left(q_{2}, \Gamma_{2}^{*}(t)\right) .
$$


We have to show that $\max \{f, g\}$ is a $\Delta$-T-function. We use without loss of generality as above that $d_{1}\left(p_{1}, p_{1}^{\prime}\right) \geq d_{2}\left(p_{2}, p_{2}^{\prime}\right)$, i.e. that $d_{m}\left(p, p^{\prime}\right)=d_{1}\left(p_{1}, p_{1}^{\prime}\right)$. In this case $d\left(\Gamma_{1}(t), \gamma_{p, p^{\prime}}(t)\right) \leq \delta^{\prime}$ for all $t \in[0, a+b]$, where $\delta^{\prime}$ only depends on $\delta$ and not on $p, p^{\prime}$. It follows that $f$ is a $\Delta$-T-function for some $\Delta$ only depending on $\delta$.

Note further that $\left.g\right|_{\left[0, a^{*}\right]}$ and $\left.g\right|_{\left[a^{*}+\epsilon, a^{*}+b^{*}\right]}$ are $4 \delta$-T-functions by Lemma 2 for every $\epsilon>0$, and hence $\left.g\right|_{\left[a^{*}, a^{*}+b^{*}\right]}$ is a $12 \delta$-T-function since the jump at $a^{*}$ is bounded by $8 \delta$.

Let $v=\left(q_{2} \cdot B_{2}\right)_{p_{2}}$, then the function

$$
[0, \infty) \longrightarrow[0, \infty), \quad t \longmapsto d_{2}\left(q_{2}, \gamma_{2}(t)\right)
$$

assumes the minimum $8 \delta$-close to the point $t=v$.

If $v>a$, hence $v \geq a^{*}-10 \delta$, then by the properties of $\delta$-T-functions $\left.g\right|_{\left[0, a^{*}+b^{*}\right]}$ is easily checked to be a $30 \delta$ - $T$-function.

Let us assume that $v<a$. Set $u:=d_{2}\left(q_{2}, p_{2}\right)-v$ and let $\tilde{\gamma}_{2}:[0, \infty) \longrightarrow X$ be a $B_{2}$-ray starting at $q_{2}$. Then $d_{2}\left(\tilde{\gamma}_{2}(u+t), \gamma_{2}(v+t)\right) \leq 8 \delta$. In particular $d_{2}\left(\tilde{\gamma}_{2}\left(u+\left(a^{*}-v\right)\right), \gamma_{2}\left(a^{*}\right)\right) \leq 8 \delta$ which implies

$$
\begin{aligned}
8 \delta & \geq\left|d_{2}\left(q_{2}, \gamma_{2}\left(a^{*}\right)\right)-\right| B_{2}\left(q_{2}\right)-B_{2}\left(\gamma_{2}\left(a^{*}\right)\right)|| \\
& =\left|d_{2}\left(q_{2}, \gamma_{2}\left(a^{*}\right)\right)-\right| B_{1}\left(q_{1}\right)-B_{1}\left(\gamma_{1}\left(a^{*}\right)\right)|| .
\end{aligned}
$$

Since $d_{1}\left(q_{1}, \gamma_{1}\left(a^{*}\right)\right) \geq\left|B_{1}\left(q_{1}\right)-B_{1}\left(\gamma_{1}(a)\right)\right|$ we obtain

$$
f\left(a^{*}\right)=d_{1}\left(q_{1}, \gamma_{1}\left(a^{*}\right)\right) \geq d_{2}\left(q_{2}, \gamma_{2}\left(a^{*}\right)\right)-8 \delta \geq g\left(a^{*}\right)-8 \delta .
$$

Since $\left.g\right|_{\left[0, a^{*}\right]}$ and $\left.g\right|_{\left[a^{*}, a^{*}+b^{*}\right]}$ are $12 \delta$-T-functions and $f$ is a $\delta^{\prime}$-T-function we see that $(g-f)_{+} \leq 20 \delta+\delta^{\prime}$ which implies that $\max \{f, g\}$ is a $20 \delta+2 \delta^{\prime}-T$-function.
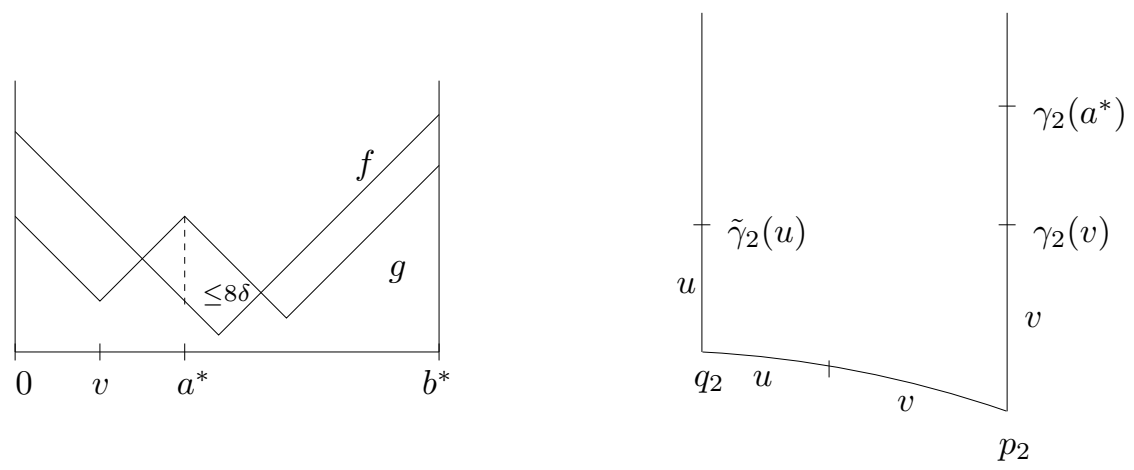

Figure 4: This figure visualizes the situation in the proof of Proposition 3.

We finally indicate how the arguments of this section have to be modified to prove Theorem 1. In the case of Theorem 1 let $B_{1}:=d\left(z_{1}, \cdot\right), B_{2}:=d\left(z_{2}, \cdot\right)$ and 
$z=\left(z_{1}, z_{2}\right)$. The $B_{i}$-rays correspond to the geodesics $\gamma_{i}=\gamma_{p_{i} z_{i}}, \gamma_{i}^{\prime}=\gamma_{p_{i}^{\prime} z_{i}}$. Let further

$$
\begin{array}{rlll}
\gamma:[0, d(p, z)] & \longrightarrow & & \gamma=\left(\gamma_{1}, \gamma_{2}\right) \\
\gamma^{\prime}:[0, d(p, z)] & \longrightarrow Y, & & \gamma^{\prime}=\left(\gamma_{1}^{\prime}, \gamma_{2}^{\prime}\right)
\end{array}
$$

$a_{i}:=\left(p_{i}^{\prime} \cdot z_{i}\right)_{p_{i}}$ and $b_{i}:=\left(p_{i} \cdot z_{i}\right)_{p_{i}^{\prime}}$.

While the definition of $\Gamma$ is analog to the one in the case of Theorem 2, the definition of $\Gamma_{p p^{\prime}}^{c}$ needs to be slightly modified in the case that $a+2 \delta>d(p, z)$. In that case just take

$$
\Gamma_{p p^{\prime}}^{c}:=\left.\left.\gamma\right|_{[0, a+\tau]} * \gamma\right|_{[0, b+\tau]}{ }^{-1},
$$

where $a \leq \tau<2 \delta$ is chosen such that $\gamma(\tau)=\gamma^{\prime}(\tau)=z$.

The proof of Lemma 8 stays valid in the case $y_{0} \neq z, \epsilon<d\left(y_{0}, z\right)$. In the case $y_{0}=z$ the result is obvious.

\section{The boundary of $Y$}

In the case of Theorem 1 it is easy to see that $\partial Y=\partial X_{1} \times \partial X_{2}$. The situation of Theorem 2 is more interesting:

We study $\partial Y$ and show that it is homeomorphic to $\partial X_{1} \wedge \partial X_{2}$.

Recall that the Busemann functions $B_{i}$ are defined as $B_{i}(x)=\lim _{t \rightarrow \infty}\left(d_{i}\left(x, \gamma_{i}(t)\right)-\right.$ $t)$, where $\gamma_{i}:[0, \infty) \longrightarrow X_{i}$ is a geodesic ray, $i=1,2$.

Let $z_{i}=\gamma_{i}(0)$ and $u_{i}=\left[\left\{\gamma_{i}(k)\right\}\right] \in \partial X_{i}, i=1,2$. Let further $z=\left(z_{1}, z_{2}\right) \in Y$ and $\gamma(t)=\left(\gamma_{1}(t), \gamma_{2}(t)\right)$. Then $\gamma$ is a ray with $\gamma(0)=z$ and clearly the Busemann function $B: Y \longrightarrow \mathbb{R}$ of this ray is $B\left(y_{1}, y_{2}\right)=B_{1}\left(y_{1}\right)=B_{2}\left(y_{2}\right)$.

Let $u:=[\{\gamma(k)\}] \in \partial Y$. Using the results of Sections 2 and 3 there exists a $\Delta$ such that the following holds:

(1) $Y$ is $\Delta$-hyperbolic,

(2) $d\left(\Gamma_{x y}^{*}(t), \gamma_{x y}(t)\right) \leq \Delta$,

(3) $d\left(\Gamma_{x y}^{*}(t), \Gamma_{x z}^{*}(t)\right) \leq \Delta \quad \forall 0 \leq t \leq(y \cdot z)_{x}$,

(4) $d\left(\Gamma_{z x}^{*}(t), \gamma(t)\right) \leq \Delta \quad \forall 0 \leq t \leq(x \cdot B)_{z}$,

(5) $\left|d_{m}(x, y)-d(x, y)\right| \leq \Delta$.

For any point $v \in \partial Y$ consider a geodesic ray $\sigma:[0, \infty) \longrightarrow Y$ such that $\sigma(0)=z$ and $v=[\{\sigma(k)\}]$. Consider the curves $\Gamma_{z \sigma(k)}^{*}$ with $d\left(\Gamma_{x \sigma(k)}^{*}(t), \sigma(t)\right) \leq \Delta$ for $0 \leq t \leq k$. By Section $3 \Gamma_{z \sigma(k)}^{*}=\left.\left.\gamma\right|_{\left[0, a_{k}^{*}\right]} * \gamma^{k}\right|_{\left[0, b_{k}^{*}\right]}{ }^{-1}$, where $\left|a_{k}^{*}-(\sigma \cdot B)_{z}\right| \leq \Delta$ and $\gamma_{i}^{k}:[0, \infty) \longrightarrow X_{i}$ is a $B_{i}$-ray with $\gamma_{i}^{k}(0)=\sigma_{i}(k)$ and $d\left(\gamma_{i}^{k}\left(b_{k}^{*}\right), \gamma_{i}\left(a_{k}^{*}\right)\right) \leq \Delta$. We distinguish two cases:

(Case 1) There exists a subsequence $\left\{a_{k_{j}}^{*}\right\}$ with $\lim _{j \rightarrow \infty}$.

Then $\lim _{j \rightarrow \infty}\left(\sigma\left(k_{j}\right) \cdot B\right)_{z}=\infty$ and thus $\liminf _{j, l \rightarrow \infty}\left(\sigma\left(k_{j}\right) \cdot \gamma(l)\right)_{z}=\infty$, which 
implies $\left\{\sigma\left(k_{j}\right)\right\} \sim\{\gamma(j)\}$, hence $\left[\left\{\sigma\left(k_{j}\right)\right\}\right]=u$. Since $\sim$ is an equivalence relation and clearly $\left\{\sigma\left(k_{j}\right)\right\} \sim\{\sigma(j)\}$ we also see that $\lim _{k \rightarrow \infty} a_{k}=\infty$.

(Case 2) $\left\{a_{k}^{*}\right\}$ is bounded.

Then it holds $b_{k}^{*} \longrightarrow \infty$. Reparameterize $\gamma_{i}^{k}:[0, \infty) \longrightarrow X_{i}$ as

$$
\bar{\gamma}_{i}^{k}:\left[a_{k}^{*}-b_{k}^{*}, \infty\right), \quad \bar{\gamma}_{i}^{k}(t)=\gamma_{i}^{k}\left(t+b_{k}^{*}-a_{k}^{*}\right) .
$$

By the discussion of Sections 2 and 3 the $\bar{\gamma}_{i}^{k}$ converge to a complete geodesic $\gamma_{i}^{*}$ with $B_{i}\left(\gamma_{i}(t)\right)=-t$ and $d_{i}\left(\gamma_{i}\left(a_{k}^{*}\right), \gamma^{*}\left(a_{k}^{*}\right)\right) \leq 8 \delta$. Clearly we have $\left[\left\{\gamma_{i}^{*}(k)\right\}\right]=u_{i}$. Let $v_{i}:=\left[\left\{\gamma_{i}^{*}(-k)\right\}\right] \in \partial X_{i} \backslash\left\{u_{i}\right\}$.

From the discussion of Case 2 it is not difficult to show that the map

$$
\begin{array}{ccc}
\partial Y \backslash\{u\} & \longrightarrow & \left(\partial X_{1} \backslash\left\{u_{1}\right\}\right) \times\left(\partial X_{2} \backslash\left\{u_{2}\right\}\right) \\
v & \longmapsto & \left(v_{1}, v_{2}\right)
\end{array}
$$

is a homeomorphism, which by the discussion of Case 1 extends naturally to a homeomorphism

$$
\partial Y \longrightarrow \partial X_{1} \wedge \partial X_{2}
$$

UNIVERSITÄT ZÜRICH, MATHEMATISCHES INSTITUT, WINTERTHURERSTRASSE 190, CH-8057 ZÜRICH, SWITZERLAND

E-mail addresses: foertsch@math.unizh.ch vschroed@math.unizh.ch

\section{References}

[BeKa] N.Benakli \& I. Kapovich, Boundaries of hyperbolic groups, preprint math.GR/0202286

[BrFa] N. Brady \& B. Farb, Filling-Invariants at Infinity for Manifolds of Nonpositive Curvature, Trans. Amer. Math. Soc., Vol. 350, Num. 8, 1998

[BriH] M. Bridson \& A. Haefliger, Metric spaces of non-positive curvature, Springer Verlag Berlin 1999

[BuBuI] D. Burago \& Y. Burago \& S. Ivanov, A course in Metric Geometry, Graduate Studies in Mathematics, Vol. 33, Amer. Math. Soc., 415pp, 2001

[F1] T. Foertsch, Bilipschitz Embeddings of Negative Sectional Curvature in Products of Warped Product Manifolds, Proc. Amer. Math. Soc. 130, 2089-2096, (2002)

[F2] The Hyperbolic Rank of Homogeneous Hadamard Manifolds, to appear in the Manuscripta Mathematicae 
[FS] T. Foertsch \& V. Schroeder, Hyperbolic Rank of Products, preprint

[L1] E. Leuzinger, Corank and Asymptotic Filling-Invariants for Symmetric Spaces, Geom. Funct. Anal., vol.10, no4, 863-873, (2000)

[L2] Bi-Lipschitz Embeddings of Trees into Euclidean Buildings, preprint

[M] C.R.F. Maunder Algebraic Topology, Cambridge University Press, 1980 